\title{
Is There A Doctor In The Emergency Department?
}

Ensuring that there are enough physicians to staff the emergency department (ED) is a major issue for hospital managers. According to a recent ICES Practice Atlas on ED Services in Ontario (Chan et al.2001), this task may become increasingly difficult. From fiscal year 1993 to 2000 , the number of physicians working in EDs declined by $21 \%$, from 2,525 to $1,987$.

One reason fewer physicians are practicing emergency medicine is because the ED is an increasingly taxing work environment. Although the per capita use of EDs has declined by $10 \%$ in the past seven years, this decline was attributable to lower ED use by children, who tend to be lower acuity cases (e.g. colds and ear infections). On the other hand, the per capita use of ED services by the elderly is rising and they present with much more complex conditions. Furthermore, 19 hospitals in Ontario out of 201 closed during the study period. As a result, ED visit volumes at the remaining hospitals rose by $10 \%$, from 19,100 to 21,000 per year.

Another warning sign is the aging ED physician workforce. In $1993,40 \%$ of ED physicians were under the age of 40 . By 2000 , this proportion dropped to $24 \%$. This phenomenon may be related to policies in the 1990s that were implemented to restrict the growth in the supply of physicians (Barer et al. 1996). These policies were targeted, perhaps unfairly, at young physicians.

A third issue concerns the existence of highly predictable peaks in ED volume. EDs see $9 \%$ more patients per day on weekends than average. The week between Christmas and New Year's is the busiest of the year, and volumes increase by up to one-third above the volumes seen on other public holidays. This raises the question of how to ensure sufficient staffing on these days.

Three questions for hospital managers to consider, in light of these findings, are as follows:

\section{Q1: What type of physician do you want to have staffing your ED?}

EDs may be staffed by general practitioners or family physicians without additional emergency training (GP/FPs); family physicians with emergency certification (CCFP(EM)s); and specialists in emergency medicine $(\mathrm{FRCP}(\mathrm{C}) \mathrm{s})$. Most ED care is still provided by GP/FPs, but most of the decline in physician numbers has been among this group.The number of CCFP(EM)s has almost doubled, and specialists remain relatively rare.

There are obvious advantages to staffing EDs with CCFP(EM) physicians. These physicians have more training and work more hours in the ED, which, in theory, may improve quality of care. However, having GP/FPs working in the ED may provide these

\begin{tabular}{lcc}
\multicolumn{3}{l}{ TABLE 1: Number of Physicians Working in EDs in Fiscal Years } \\
1993 and 2000 \\
Specialty & 1993 & 2000 \\
GP/FPs & 2,258 & 1,631 \\
CCFP(EM) Physicians & 145 & 275 \\
Emergency Medicine & 42 & 46 \\
Other & 80 & 36 \\
\hline TOTAL & 2,525 & 1,987 \\
Note: physicians working in non-fee-for-service settings excluded.
\end{tabular}

physicians with the opportunity to work in a challenging environment that encourages peer interaction and may help maintain clinical skills. Furthermore, being able to draw on a larger pool of GP/FPs who do part-time ED work may provide greater flexibility in call scheduling and make the hospital less prone to staffing crises should one physician leave.

Hospitals and physicians need to consider which model they prefer. If they want more specialized ED physicians, then training spots in emergency medicine need to be dramatically increased. If the GP/FP model is preferred, then training programs must ensure that FP residents get sufficient exposure to the ED. Hospitals also must consider whether there are local hospital policies in place that may have discouraged GP/FPs from participating in the provision of hospital care.

\section{Q2: Are there local issues that have resulted in the elderly coming to EDs more often than in the past?}

Future ICES research will explore this question further. In the meantime, hospital managers can start making inquiries locally. Are the elderly visiting the ED because of insufficient home care? Do they have someone to call if a complication in treatment arises? Are discharge plans adequately developed? Engaging local stakeholders in such a debate may help to identify opportunities to have the elderly cared for in the community, rather than in the ED.

\section{Q3: In your hospital, do you vary physician staffing according to anticipated peak periods, or vary physician remuneration according to workload?}

The highly predictable nature of peaks in patient volume during holidays behooves ED planners to be well prepared for them. Strategies may include having extra physicians present in the ED. Financial incentives may be necessary in order to encourage physicians to work during these particularly stressful days, particularly where physicians are paid a fixed hourly rate, regardless of patient volume.

Not all peak days are predictable, however, and hospitals 
need to consider how to best handle unanticipated fluctuations. This may include a system of second on-call, where physicians may be called in during periods of excessive workload. Hospitals may also wish to develop criteria indicating when the on-call physician should be brought in (e.g. if waiting times exceed a certain threshold).

Dealing with the issues raised in this ICES Atlas is easier said than done. Addressing the above concerns requires sufficient resources, such as hospital budgets to pay for additional staff. Planning for peaks is particularly difficult if a community is already facing a shortage of physicians and nurses. Although there are opportunities for hospital planners to develop more creative solutions, Ministries of Health also have a responsibility to ensure the system has sufficient funding to meet these challenges.

\section{References}

Chan B.T.B., M.J.Schull and

S.E. Schultz. 2001. Atlas Report:

Emergency Services in Ontario,

1993-2000. Toronto: Institute for

Clinical Evaluative Sciences.

Barer M.L., J. Lomas and C.Sanmartin. 1996."Re-minding our Ps and Qs: Medical Cost Controls in

Canada." Health Affairs 15(2): 216-34.

Ben Chan is a Senior Scientist at the Institute for Clinical Evaluative Sciences in Toronto. He also practices part-time as a locum family physician and has worked in over 40 EDs across Canada.

For more information see www.ices.on.ca

\section{What do you do with employees harassing employees?}

\section{Let the HRBoard provide you with solutions!}

Scenario: You have abusive and uncooperative employees. They are bullies. You know you need to resolve this. Fast. But no policies, no programs, and no manuals deal specifically with your case. Worse, you have no researcher to turn to and your own time is booked solid. Where do you turn?

Think of us as your researchers.

Response: We will sort out what others have done using criteria established by you and your associates. We'll look for case studies, strategies and road maps with benchmarks. We'll look for best practices. Then, we'll make the information available directly to you.

\section{How do we do that?}

Methodology: The publishers have set up the HRBoard - an elite compendium of members who share intellectual resources, reports, findings, proceedings and other published or unpublished information related to HR management. Think of it as on-demand, just-in-time learning. The board is for HR executives in organizations providing healthcare services. Members share their concerns and tell us what they need. We help to define the issues and search for proven and practical answers. We will go to peer organizations, other industries, research institutions, governments and consultants. If the answers are out there, we will find them for you. To the point and fast.

Best of all, there are no fees.

Contact the publisher for more information. You can start tomorrow.

\section{HRBOARD}

260 Adelaide Street East, No.8, Toronto, ON M5A 1N1

For more information write:

Anton Hart at ahart@longwoods.com

Or call him at 4168649667 